\title{
A new attachment hood (Nanoshooter) for transnasal endoscopic submucosal dissection of the hypopharynx
}

Endoscopic submucosal dissection (ESD) in the pharyngeal region is considered difficult. When ESD is carried out transorally for a tumor in the pharynx, the patient must be sedated [1]. However, we have carried out, without sedation, ESD with a transnasal endoscope and our novel endoscopic attachment hood for this area. There is evidence that our method may be associated with reduced incidence of hemorrhage and perforation [2]. Here, we report on the use of ESD in the treatment of pharyngeal dysplasia.

We have recently developed an attachment hood (TOP-16561, Nanoshooter) with a waterjet channel as well a suction channel for removing the residual water from the hood ( $\bullet$ Fig. 1), which we use with a transnasal endoscope (EG-530N2, Fujifilm, Tokyo, Japan; this is the only transnasal endoscope that can be used with a high-frequency electrosurgical system). The dysplastic lesion appeared as a brownish area, and irregular vessels were evident with a narrow-band imaging optical zoom endoscope ( Fig.2). We used our original precutting knife for resection and ICC200 (ERBE USA, Marietta, Georgia, USA) with the conventional settings. Without use of sedation, the endoscope with the Nanoshooter attached to it was inserted transnasally and a $1: 1$ mixture of lidocaine and $0.4 \%$ hyaluronic acid [3] was injected submucosally ( $\bullet$ Fig. 3). After lifting the lesion, complete resection was carried out. After resecting the mucosal layer and trimming around the lesion, we used a snare to resect the lesion, reducing the procedure time ( $\bullet$ Fig.4) to approximately 30 minutes in total ( $\bullet$ Video 1$)$.

\section{Video 1}

Illustration of the procedure for transnasal endoscopic submucosal dissection (ESD) with the Nanoshooter.
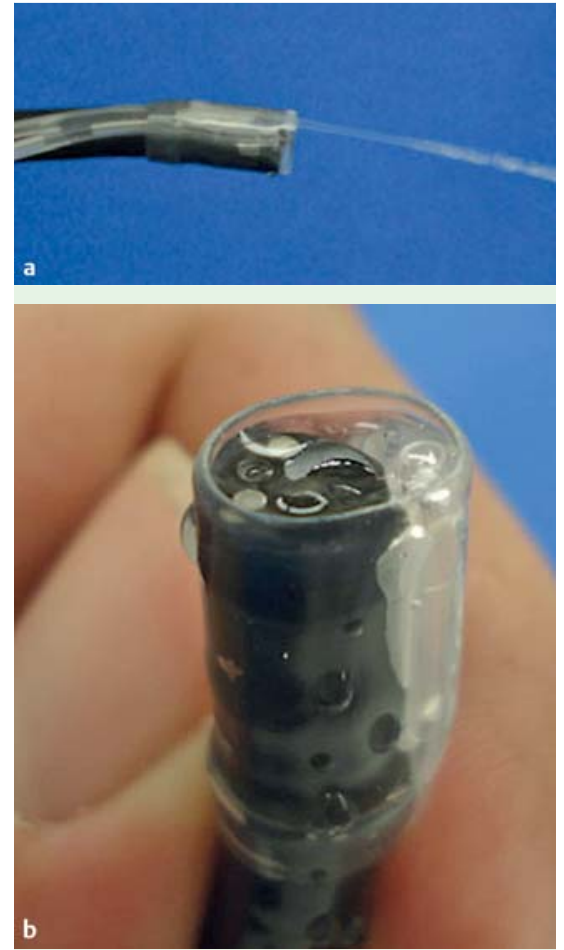

Fig. 1 The Nanoshooter: a water being discharged from a waterjet channel; and $\mathbf{b}$ the waterjet and suction channels.

Pharyngeal ESD was easily carried out with our method because the pharynx could be directly observed from straight ahead. Moreover, the procedure was done without sedation and with causing little pain to the patient. This method shows potential for esophageal ESD. In the near future, it will be possible to use this treatment method with the Nanoshooter to enable transnasal ESD, without sedation, for neoplasms of the upper gastrointestinal tract in elderly and high-risk patients with cardiopulmonary disease.

Endoscopy_UCTN_Code_TTT_1AO_2AG

Competing interests: None
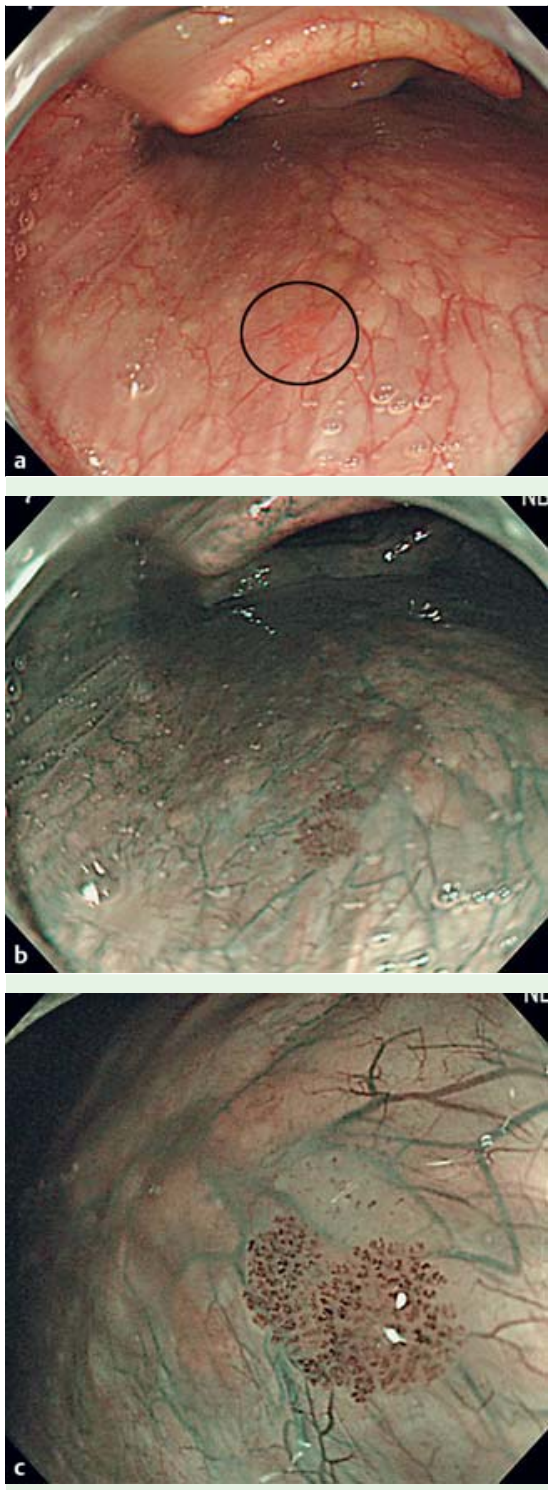

Fig. 2 Dysplastic lesion in the pharynx. a The dysplastic lesion (encircled). b Lesion appearing as a brownish area with narrow-band imaging optical zoom endoscopy. c Irregular vessels in the area. 

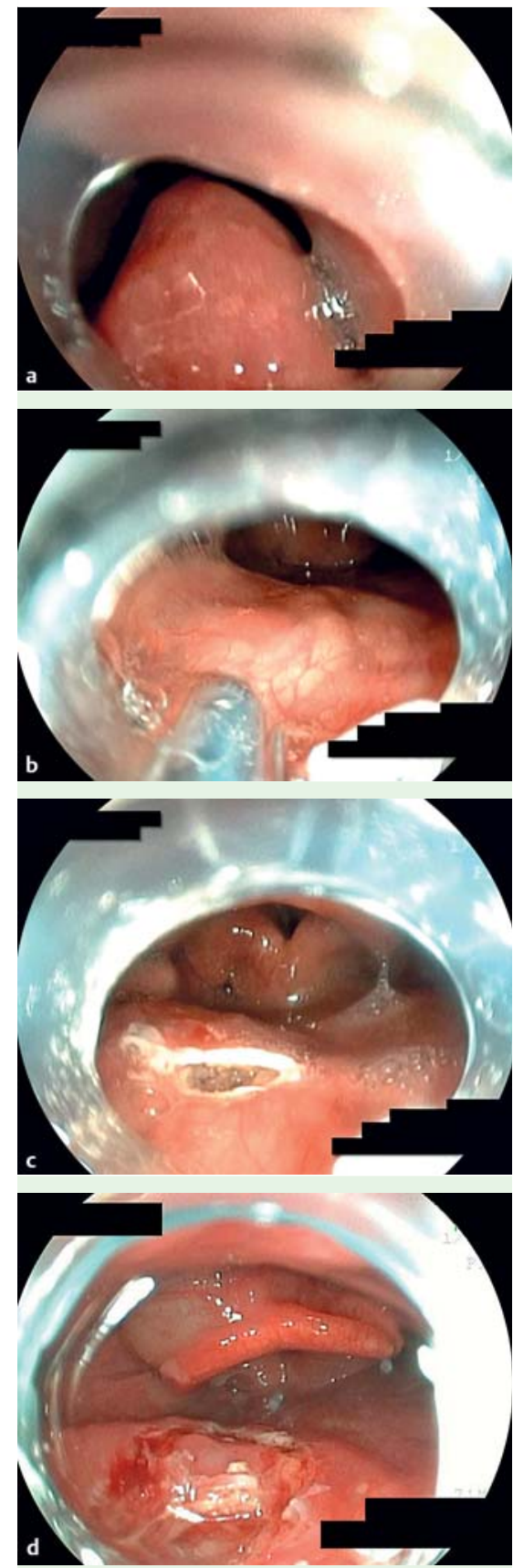

Fig. 3 Endoscopic submucosal dissection (ESD) using a transnasal endoscope with the Nanoshooter: part 1. a The endoscope with the Nanoshooter attached to it was inserted transnasally. $\mathbf{b}$ A mixture of lidocaine and hyaluronic acid was injected into the submucosal layer. c The mucosal layer was incised. $\mathbf{d}$ Finally, an incision was made around the lesion.


Fig. 4 Endoscopic submucosal dissection (ESD) using a transnasal endoscope with the Nanoshooter: part 2. a A snare was used to start resecting the lesion. $\mathbf{b}$ The dysplastic lesion was completely resected. c The resected dysplastic lesion.

\section{Masakatsu Nakamura ${ }^{1}$, Hisakazu}

Shiroeda ${ }^{1}$, Tomomitsu Tahara², Tomoyuki Shibata ${ }^{2}$, Tomiyasu Arisawa ${ }^{1}$

1 Department of Gastroenterology, Kanazawa Medical University, Daigaku, Uchinada-machi, Ishikawa, Japan

${ }^{2}$ Department of Gastroenterology, Fujita Health University, Dengakugakubo, Kutsukake-cho, Toyoake, Aichi, Japan

\section{References}

1 Iizuka T, Kikuchi D, Hoteya $S$ et al. Endoscopic sub-mucosal dissection for treatment of mesopharyngeal and hypopharyngeal carcinomas. Endoscopy 2009; 41: 113-117

2 Nakamura M, Shibata T, Tahara T et al. Usefulness of transnasal endoscopy where endoscopic submucosal dissection is difficult. Gastric Cancer 2011; 14: 378-384

3 Yamamoto H, Koiwai H, Yube T et al. A successful single-step endoscopic resection of a 40 millimeter flat-elevated tumor in the rectum, endoscopic mucosal resection using sodium hyaluronate. Gastrointest Endosc 1999; 50: $701-704$

\section{Bibliography}

Dol http://dx.doi.org/

10.1055/s-0033-1344824

Endoscopy 2013; 45: E387-E388

(c) Georg Thieme Verlag KG

Stuttgart · New York

ISSN 0013-726X

\section{Corresponding author}

Tomiyasu Arisawa

Department of Gastroenterology

Kanazawa Medical University

1-1, Daigaku

Uchinada-machi

Ishikawa 920-0293

Japan

Fax: +81-76-2860892

tarisawa@kanazawa-med.ac.jp 